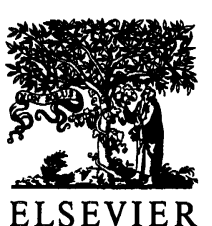

Journal of

Back and

Musculoskeletal

ELSEVIER

Journal of Back and Musculoskeletal Rehabilitation 9 (1997) 1

Rehabilitation

\title{
From the Editor
}

This issue of the Journal of Back and Musculoskeletal Rehabilitation contains the proceedings of the 25th Anniversary Meeting of the Society for Back Pain Research and is brought to you with the cooperation of the Royal Society of Medicine in London.

The number of individuals with chronic back pain continues to rise in the United States, costing billions of dollars in medical care, lost work days, and disability pay. Yet, despite its increasing medical and economic impact, many aspects of pain assessment and treatment remain unclear. For this reason, we thought it would be beneficial to our readers to present the complexity of back pain from an international perspective. As you can see, the controversy surrounding chronic back pain is not limited to the United States. Questions persist as to what factors should be involved in the assessment of chronic back pain, in disability determination and in assigning appropriate treatment protocols.

Unlike previously published proceedings, which presented papers based on talks given, this issue contains actual talks from the meeting. We have chosen to present it this way in an effort to maintain the atmosphere in which the meeting took place. In particular, the debate at the end of the meeting is presented in its original 'no holds barred' format, giving readers a clear picture of the current controversies in back pain today.

We hope you find this issue interesting and educational. As the debate continues over how to properly assess chronic back pain, return to work and ongoing rehabilitation efforts, it is imperative that we remain open to methods being utilized worldwide in trying to answer these and other questions.

As always, we welcome your comments regarding this and other issues of the Journal.

Karen S. Rucker, MD 